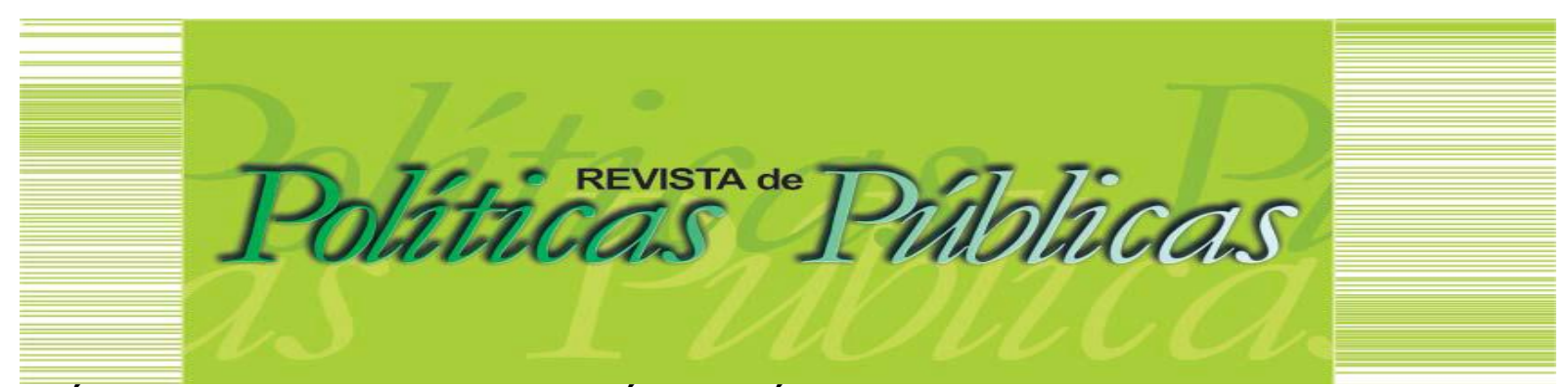

\title{
ÍNDICE DE EFETIVIDADES DE POLITIICAS PÚBLICAS DE SEGURANÇA ALIMENTAR PARA AGRICULTURA FAMILIAR NO SUDOESTE PAULISTA
}

\author{
Daiane Roncato Cardozo 1 \\ Luiz Fernando de Orianni e Paulillo2 \\ Luiz Manoel de Moraes Camargo Almeida ${ }^{3}$, \\ Herick Fernando Moralles ${ }^{4}$
}

\section{Resumo}

O trabalho tem objetivo de avançar na construção de um novo índice de efetividades de políticas públicas (EPP) de segurança alimentar (SA) para agricultores familiares da região sudoeste paulista. Foi utilizada a técnica de análise fatorial a partir de um conjunto de variáveis com aderência teórica e metodológica à SA dos agricultores familiares, pressupondo que a Escala Brasileira de Insegurança Alimentar (EBIA) possui limitações analíticas. Constata que uma elevação de $1 \%$ no EPP acarreta um aumento de aproximadamente $0,51 \%$ no índice de SA para a agricultura familiar; e $1 \%$ na renda total eleva em $0,26 \%$ o indicador de SA. Conclui que de forma similar, os indivíduos com acesso ao mercado institucional possuem um nível de SA $15 \%$ acima da média. A aplicação e a análise do índice UFSCar mostraram que também é possível comparar impactos para agricultores familiares de outras regiões no Brasil.

Palavras-chave: Desenvolvimento territorial. Escala Brasileira de Insegurança Alimentar. Políticas públicas. Renda agrícola. Análise fatorial ortogonal.

\section{INDEX OF EFFECTIVENESS OF PUBLIC POLICIES OF FOOD SECURITY FOR FAMILY FARMING IN SOUTHWEST OF SÃO PAULO}

\section{Abstract}

The work aims to advance the construction of a new index of public policies effectiveness (PPE) food security(FS) for family farmers in the southwest region of São Paulo. The factor analysis technique was used from a set of variables with theoretical and methodological adherence to the FS of family farmers, assuming that the Brazilian Food Insecurity Scale(BFIS) has analytical limitations. Notes that a $1 \%$ increase in the PPE leads to an increase of approximately $0.51 \%$ in the FS to family farming; and $1 \%$ in total income increases $0,26 \%$ the FS indicator. It concludes that similarly, individuals with access to the institutional market have an FS level 15\% above the average. The application and analysis of the UFSCar index showed that it is also possible to compare impacts for family farmers from other regions in Brazil.

Keywords: Territorial development. Brazilian Food Insecurity Scale. Public policies. Agricultural income. Orthogonal factorial analysis.

Artigo recebido em: 15/04/2020 Aprovado em: 25/10/2020 DOI: http://dx.doi.org/10.18764/2178-2865.v24n2p819-838

\footnotetext{
${ }^{1}$ Administradora...Doutora em Alimentos e Nutrição pela Faculdade de Ciências Farmacêuticas da UNESP Campus de Araraquara-SP.. Professora colaboradora do PPG em Desenvolvimento Territorial e Meio Ambiente da UNIARA e Professora Substituta da área de Gestão do IFSP Campus Boituva E-mail: daianecardozo@msn.com

2 E-mail: dlfp@ufscar.br

3 Engenheiro de Produção Agroindustrial. Doutor em Sociologia pela Universidade Estadual Paulista Júlio de Mesquita Filho. Professor do Centro de Ciências da Natureza do Campus Lagoa do Sino da Universidade Federal de São Carlos (UFSCar). Professor e Pesquisador dos Programas de Pós Graduação em Gestão de Organizações e Sistemas Públicos da UFSCar e de Desenvolvimento Territorial e Meio Ambiente da Universidade de Araraquara-SP Graduação em Engenharia pela Universidade Federal de São Carlos.. Diretor do Centro de Ciências .Exatas e de Tecnologia - UFSCar São Carlos. Diretor do Centro de Ciências da Natureza - UFSCar. E-mail: manoel77@yahoo.com.br.

${ }^{4}$ Economista. Doutor em Engenharia de Produção pela Universidade de São Paulo. Professor com experiência na área de Modelos e Métodos Econométricos e Estatísticos, atuando principalmente nos seguintes temas de investigação: E-mail: herickmoralles@dep.ufscar.br
} 


\section{INTRODUÇÃO}

A Segurança Alimentar e Nutricional (SAN) atua em função de cinco grandes eixos de valores ligados à saúde, higiene, autenticidade, meio ambiente e solidariedade da população. A solução consensual entre os países participantes da Conferência de Roma de 1996 da Food and Agriculture Organization of the United Nations (FAO/ONU) abriu um novo leque de problemas e desafios na agenda das políticas públicas de SAN no mundo.

Os eixos autenticidade, meio ambiente e solidariedade da SAN chamaram a atenção para os limites dos programas generalizados de políticas públicas. Assim, indicadores de efetividade de políticas públicas de SAN não devem ignorar as delimitações regionais dos programas, que focam um primeiro conjunto de especificidades econômicas, sociais e culturais. Isso importa no Brasil, uma vez que governos recentes experimentaram delimitações territoriais para programas de SAN como territórios da cidadania, territórios rurais, bacias hidrográficas, municípios, entre outros (PAULILLO; PESSANHA, 2002).

A evolução de conceitos e padrões de SAN no mundo traz a necessidade de novos descritores em índices capazes de registrar o estágio atual das sociedades na busca do combate à fome, o que pode ser possibilitado através da construção de uma série de variáveis úteis para aprimorar políticas e programas de SAN (ALMEIDA et al., 2018).

As políticas públicas consistem em decisões e ações do governo que produzem efeitos específicos, de acordo com interesses de um determinado campo de forças, e enfatizam seu papel na solução - nem sempre adequada - de problemas da sociedade (SOUZA, 2006). Um dos aspectos mais importantes da avaliação da ação pública é exatamente a identificação dos seus efeitos em função do referencial escolhido, sendo, no caso deste artigo, a avaliação comparativa da SAN de agricultores familiares do território sudoeste paulista, região caracterizada por sujeitos que vivenciaram programas de SAN, que participaram de mercados institucionais de alimentos e do Programa de Aquisição de Alimentos (PAA), usaram crédito para custeio ou capital nas últimas safras e que também puderam receber assistências técnicas rurais(Ater).

Quando o foco do programa de política pública estiver na categoria econômica (agricultor familiar, trabalhador rural, etc.), as definições de cada eixo de atuação em SAN serão mais específicas que as soluções dos programas generalizantes como, por exemplo, o Programa Bolsa Família do governo brasileiro. A descoberta da especificidade pode ser facilitada quando a perspectiva territorial for adotada, isto é, a delimitação territorial passa a importar para a gestão da política pública de SAN, seja na perspectiva de território-zona seja na de território-rede. Com isso, tem-se buscado consolidar uma construção metodológica de avaliação dos entraves e das efetividades sociais de políticas 


\section{ÍNDICE DE EFETIVIDADES DE POLÍTICAS PÚBLICAS DE SEGURANÇA ALIMENTAR PARA AGRICULTURA FAMILIAR NO SUDOESTE PAULISTA}

públicas de SAN e de programas de transferência de renda no campo do governo federal sob a gestão dos municípios e seus contornos específicos em diferentes territórios.

Nesse sentido, o objetivo principal deste artigo foi avançar na construção de um novo índice de efetividades de políticas públicas (EPP) de SAN para agricultores familiares da região sudoeste paulista. $O$ índice de EPP de SAN para a agricultura familiar da região do Campus Lagoa do Sino da Universidade Federal de São Carlos (UFSCar) ${ }^{1}$ pode significar um avanço metodológico para ir além da compreensão dos estágios de insegurança alimentar do agricultor familiar. Almeida (2009) já colocou sobre a necessidade de considerar as especificidades de uma realidade territorial rural baseada no método perceptivo familiar, a partir da Escala Brasileira de Insegurança Alimentar (EBIA)².

0 índice apresentado neste estudo adota uma delimitação territorial ${ }^{3}$ que abrange 40 municípios das regiões de Itapeva, Itapetininga e Avaré, Estado de São Paulo, sendo parte relevante os municípios de um arranjo institucional da região de Itapeva, denominado Consórcio de Segurança Alimentar e Desenvolvimento (Consad) ${ }^{4}$ do sudoeste paulista.

No caso dessa delimitação territorial, os municípios foram agrupados porque estão na classificação mais baixa do Índice de Desenvolvimento Humano (IDH) da macrorregião de Sorocaba e, coincidentemente, do Estado de São Paulo. Desse modo, o trabalho considera que as dificuldades e perenidades iniciais dos agricultores familiares deste território são as mesmas, porque os indicadores clássicos de riqueza, educação e saúde desses municípios estão no mesmo nível de bem-estar social.

Neste estudo, foi construído e mensurado um índice de efetividades do PAA para os agricultores familiares assentados do território analítico delineado, que adquire características específicas para os atores, conforme a metodologia da UFSCar, por meio da técnica de rotação fatorial ortogonal (VARIMAX), para a composição do índice a partir de um conjunto de variáveis e cruzamentos entre tais variáveis, que têm aderência teórica e metodológica com os objetivos do programa e com a SAN dos agricultores. Os resultados dos índices UFSCar de SAN e de EPP foram comparados para uma análise de suas variáveis, buscando identificar se as políticas públicas têm efetiva contribuição para a SAN dos agricultores familiares analisados.

A hipótese principal deste trabalho é que o índice UFSCar de SAN corrobora a metodologia EBIA e avança no entendimento de que outras variáveis devem ser agrupadas para aumentar as efetividades das políticas públicas de SA territorial no Brasil. Partiu-se do pressuposto que a EBIA tem limitações analíticas em níveis meso e micro para responder pelas condições de SA desses agricultores e suas famílias. Isso acontece por causa das especificidades econômicas e sociais do ator e das dinâmicas territoriais envolvidas no cotidiano do produtor, propiciando uma visão limitante para a avaliação mais aprofundada de suas condições de SA. 
2 NEOINSTITUCIONALISMO E SEGURANÇA ALIMENTAR: reciprocidade e confiança nas construções de redes por meio de políticas públicas

Este trabalho está amparado em tais características, destacados em autores da abordagem do Neoinstitucionalismo Sociológico (NIS). O NIS, em linhas gerais, se contrapõe ao pensamento racionalista (do neoinstitucionalismo econômico e também da economia neoclássica), estabelecendo seu foco em aspectos culturais e cognitivos, bem como nas relações sociais individuais ou grupais. Segundo Hall e Taylor (1996), o NIS surge com um enfoque de natureza cognitiva, numa forte relação com a teoria das organizações, postulando que as normas e procedimentos não surgem como uma resposta eficiente da sociedade para a execução de tarefas, mas sim como práticas culturais (cerimônia e mitos) que se assemelham, constituindo-se em um processo denominado isomorfismo, ou seja, a tendência das organizações tornarem-se semelhantes ao longo do tempo.

Como um produto da evolução capitalista contemporânea, a incidência de formas mais sofisticadas de cooperação e relacionamento em redes (locais ou supralocais) chama a atenção de pesquisadores para a importância do estudo destas formas e suas implicações em termos de desempenho e competitividade. Estrutura-se assim, especialmente a partir dos anos 90, uma abordagem baseada no conceito de rede voltada para fenômenos caracterizados por relacionamentos cooperativos indutores de interdependências entre os agentes, que geram a necessidade de formas de coordenação coletiva das suas atividades.

Apresentam-se novas possibilidades de atuação empresarial, dentre as quais, a operação através de redes de cooperação, com seus integrantes atuando de forma independente, mas coordenada (por vezes temporária), explorando complementaridades mútuas e compartilhando informações, riscos, recursos e produção. Estas possibilidades encontram grande relevância quando se trata de redes de políticas públicas como as de SAN.

Considera-se que, fundamentalmente, o processo de estruturação e evolução das redes de políticas de SAN envolve não apenas fatores econômicos, tais como aumento de escala de compras para redução de custo médio de aquisição de alimentos, melhoria de efetividade do programa ou mesmo redução de custos de relacionamento. Ainda que os fatores econômicos não possam ser descartados, existem também componentes sociais e institucionais tais como a busca da legitimidade, a importância da homogeneidade do grupo, os mecanismos de coordenação em redes, as intensidades das relações e a questão da confiança, reciprocidade e pertencimento para a manutenção da rede.

Em alguns momentos, o arranjo em rede torna-se um fator de sobrevivência e sucesso. Devido à crescente complexidade das políticas públicas, os atores coordenadores encontram dificuldades em absorver todas as capacidades (em todas as áreas) e implementação de um programa 


\section{ÍNDICE DE EFETIVIDADES DE POLÍTICAS PÚBLICAS DE SEGURANÇA ALIMENTAR PARA AGRICULTURA FAMILIAR NO SUDOESTE PAULISTA}

(como um PAA local). A cooperação possibilita um mecanismo para superar esta dificuldade. As redes se apresentam, neste sentido, como um arranjo composto de várias capacidades, proporcionando maior flexibilidade e permitindo que seus integrantes se concentrem em suas competências essenciais e que, ao mesmo tempo, atinjam economias de escala e escopo. Por outro lado, quaisquer arranjos cooperativos colocam uma série de desafios e obstáculos na sua construção e gestão. O compartilhamento de conhecimentos, informações e experiências, necessita, portanto, da construção cuidadosa da integração bem como da superação da desconfiança.

Um aspecto da ação cooperativa na forma redes de políticas públicas é a existência de mecanismos de coordenação formais e informais. Os mecanismos de coordenação se apresentam como um aspecto importante do funcionamento do programa em rede, sendo que, especialmente nos casos analisados, há uma nítida combinação entre formalidade e informalidade. Neste trabalho, encontram-se tanto aqueles mecanismos de coordenação mais sintonizados com o controle e formalismo, tais como o estatuto da rede, atas de reuniões e regras de controle, como também mecanismos informais, baseados em confiança e reciprocidade, que tanto ajudam na efetividade do programa para o público-alvo (agricultor familiar).

As abordagens no campo de conhecimento denominado políticas públicas, assim como instituições, regras e modelos que envolvem sua criação, implementação e avaliação, tornaram-se mais salientes nas últimas décadas. Souza (2006), em sua revisão da literatura, buscou mapear os diferentes conceitos e modelos de formulação e análise de políticas públicas, fazendo uma introdução aos principais fatores que registraram nas últimas décadas a importância deste campo de conhecimento: a adoção de políticas restritivas de gastos; as novas visões sobre o papel dos governos e a substituição das políticas keynesianas pela ênfase no ajuste fiscal; além da falta de políticas públicas que promovam o desenvolvimento econômico e a inclusão social em grande parte da população em países em desenvolvimento, em especial os da América Latina.

Para Bucci (2002), as políticas públicas são um conjunto de programas de ação governamental visando coordenar os meios à disposição do Estado e as atividades privadas, para a realização de objetivos socialmente relevantes e politicamente determinados. Segundo Souza (2006), esse campo do conhecimento é tão amplo e complexo que pode ser dividido em subáreas das políticas públicas: análise de políticas, que tem como questão fundamental "o que se deve fazer", ou seja, determinar a melhor política dado um determinado problema, relação entre eficiência e efetividade; análise de processos, na qual implica responder "como e por que as políticas se desenvolvem" ou "por que algumas políticas se mantêm e outras não ao longo do tempo"; o processo decisório, relacionado ao incrementalismo 5 e tem como a questão "quem toma as decisões" e "como elas são escolhidas"; a implementação de políticas, que procura responder o "por que uma política falha ou não" e "como uma 
decisão pode ser transformada numa ação"; e avaliação de políticas, que apresenta questões como "o que foi feito" ou "quais impactos ou efeitos a política obteve, qual foi a eficiência, eficácia, entraves, dentre outros". Esta última é a de qual se trata no presente trabalho.

O conceito de avaliação adotado aqui se refere à análise de processos, produtos ou resultados de atividades ou ações desenvolvidas - programas ou políticas públicas (policy). A avaliação é uma forma de pesquisa social aplicada, sistemática, planejada e dirigida. Destina-se a identificar, obter e proporcionar de maneira válida e confiável, dados e informações suficientes e relevantes para apoiar um juízo sobre o mérito e o valor dos diferentes componentes de um programa (tanto na fase de diagnóstico, programação e execução) ou de um conjunto de atividades específicas que se realizam, foram realizadas ou se realizarão, com o propósito de produzir efeitos e resultados concretos; comprovando a extensão e o grau em que se deram estas conquistas, de tal forma que siva de base ou para uma tomada de decisões racional e inteligente entre cursos de ação, ou para solucionar problemas e promover o conhecimento e a compreensão dos fatores associados ao êxito ou fracasso de seus resultados (AGUILLAR; ANDER-EGG, 1994 apud BELLONI; MAGALHÃES; SOUZA, 2001).

O acompanhamento dos processos pelos quais elas são implementadas e a avaliação de seu impacto e efeito sobre a situação existente deve ser permanente. Assim, governo e sociedade devem fomentar o diálogo e a negociação entre os diferentes atores setoriais, avaliando e transformando qualitativamente os processos de gestão não apenas para a efetividade dessas políticas, mas também para o alcance de objetivos mais amplos orientados ao desenvolvimento econômico e social.

A SAN vai além do acesso à renda porque deve ser reconhecida como um recurso fundamental de inclusão social. Desde 1996, com a Conferência Mundial sobre SAN realizada pela FAO/ONU em Roma, as políticas de SAN devem responder pela produção, distribuição, acesso e consumo de alimentos e devem estar ligados a valores fundamentais da população: saúde, higiene, meio ambiente, autenticidade e solidariedade. Todos esses valores são socialmente construídos e compartidos na sociedade, nos mercados e nas políticas públicas.

A evolução de conceitos e padrões de SA no mundo exige novos descritores em índices que possam registrar o estágio atual da humanidade para combater a fome. Isso pode ocorrer por meio da construção de uma série de variáveis úteis para aprimorar políticas públicas de SAN. Com foco no tipo de ator que se pretenda atingir, e descritores específicos para mostrar a SA do sujeito, um programa de SAN territorial estará mais apto a alcançar os seus objetivos. Abrem-se, portanto, maiores possibilidades para esses programas atingirem maior efetividade, pois podem estar pautados por indicadores mais específicos ou próximos da realidade daquele ator. 


\section{ÍNDICE DE EFETIVIDADES DE POLÍTICAS PÚBLICAS DE SEGURANÇA ALIMENTAR PARA AGRICULTURA FAMILIAR NO SUDOESTE PAULISTA}

A agricultura familiar é um bom exemplo. Quando uma região sofre com um recuo médio nas cotações de preços de commodities agrícolas, como aconteceu em 2014 na América Latina e Caribe (queda de 5,5\%), olhar unicamente a renda auferida pelo agricultor pode afetar a efetividade de um programa de SAN neste campo econômico. Movido somente pelo nível de renda auferido pelo agricultor, o programa encontra limites para inovar. Novos estímulos devem ser dados pelas políticas de SAN para agricultores familiares quando o indicador de preços agrícolas num continente cai sucessivamente por anos, como foi o indicador de preços da FAO para América Latina e Caribe (SILVA FILHO, 2005).

Já que está muito voltado para seu território produtivo, o agricultor familiar pode alcançar ou melhorar a sua SA caso tenha diversidade de renda, pluriatividade econômica, autoconsumo familiar e participação relevante em alguma organização social - que the permita alcançar informação e melhorar a interação produtiva e social e entre numa rede de decisão política. São exemplos que podem servir de descritores de um índice de efetividade de política pública de SAN para agricultura familiar no Brasil.

Políticas públicas e SA são temas que se retiram do campo de políticas compensatórias e mesmo dos exclusivamente setoriais. Há um campo de criação de políticas com ênfase no médio e no longo prazo que se justifica pelo interesse de articulação com estratégias de desenvolvimento, notadamente as políticas criadoras de oportunidades de trabalho e renda para os pequenos e médios empreendimentos agrícolas e agroindustriais, orientadas por um planejamento agroalimentar.

Importa não restringir a abordagem de políticas públicas ao tratamento da ação estatal em si, mas que requer uma formulação menos convencional sobre a natureza e os papéis dos mercados, isto é, dos agentes privados e dos mecanismos de governança econômica das diversas atividades e segmentos produtivos envolvidos.

O trabalho avança nas incorporações de descritores e índices que representem um avanço da agenda de efetivar e atualizar políticas e programas de SAN no Brasil. A inclusão de fatores complementares à renda familiar do agricultor, como a inserção da família em algum programa de SAN, o destino da produção agrícola para o mercado institucional, o uso de crédito para custeio ou capital nas últimas safras, o recebimento de assistência técnica rural e o acesso regular do agricultor ao PAA podem ajudar neste avanço.

3 METODOLOGIA: população e instrumentos de pesquisa

O território analisado apresenta grande participação de estabelecimentos rurais de agricultura familiar, sendo 9.989 de um total de 14.163 estabelecimentos (71\%), ou seja, um percentual 
superior aos $65 \%$ do Estado de SP, embora seja inferior ao nacional, que apresenta $88 \%$ do total de estabelecimentos rurais familiares. Na região analisada, há grande diversidade produtiva, com destaque para as culturas do algodão, feijão, tomate, trigo, batata e também frutas como pêssego, maçã, etc. Este já é mais um ponto que clama atenção para as especificidades do agricultor familiar e as políticas públicas ou programas criados para desenvolver SA local.

Para o cálculo do tamanho amostral dos agricultores familiares consideraram-se as três microrregiões pertencentes à macrorregião de Sorocaba/SP: Itapetininga (região A), Itapeva (região B) e Avaré (região C), além do fato de que todos os subgrupos são mutuamente exclusivos e compõem a mesma população, o que implica a utilização da técnica de amostragem estratificada proporcional, conforme Cohran (1953). Ao todo foram coletadas informações relativas a 70 indivíduos para a amostra de agricultores familiares, o que satisfaz o tamanho total da amostra para o valor do cálculo amostral com erro igual a $10 \%$.

O cálculo do tamanho amostral baseia-se em uma questão chave do tipo dicotômica em cada questionário aplicado, possuindo o contexto de maior variabilidade possível, ou seja, $50 \%$ de resposta para cada categoria $(p=0,50)$. Para exposição dos resultados, utilizou-se 0 erro amostral de $5 \%(B=0,05)$ e $10 \%(B=0,10)$, e um nível de significância correspondente a $5 \%$ $(Z=1,96)$. A expressão do cálculo do tamanho amostral para o caso de amostragem aleatória estratificada é dada abaixo (SILVA, 2001):

$$
n=\frac{\sum_{i=1}^{L}\left(\frac{N_{i}^{2} p_{i}\left(1-p_{i}\right)}{w_{i}}\right)}{N^{2}\left(\frac{B}{z_{\alpha / 2} / 2}\right)^{2}+\sum_{i=1}^{L} N_{i} p_{i}\left(1-p_{i}\right)}, \text { onde } w_{i}=\frac{N_{i}}{N}, n_{i}=w_{i} n \text { e } L=5 \text {. }
$$

Todo o procedimento assume que as unidades amostrais sejam coletadas aleatoriamente, de modo que os resultados são exibidos na Tabela 1.

Tabela 1 - Resultado do cálculo do tamanho amostral para erro igual a 5 e 10\%

\begin{tabular}{ccccc}
\hline Região & População & $\mathbf{N}$ & $\mathbf{n}(\boldsymbol{B}=\mathbf{0 , 0 5})$ & $\mathbf{n}(\boldsymbol{B}=\mathbf{0 , 1 0})$ \\
\hline A & Agricultura Familiar & 7603 & 42 & 11 \\
B & Agricultura Familiar & 9091 & 50 & 13 \\
C & Agricultura Familiar & 811 & 5 & 2 \\
\hline
\end{tabular}

Fonte: Adaptado de Instituto Brasileiro de Geografia e Estatística (IBGE, 2010).

A pesquisa de campo foi realizada por meio de entrevistas semiestruturadas, as quais foram norteadas por um questionário formado por questões abertas e fechadas com o objetivo de caracterizar os agricultores familiares através de um olhar da AS, tratada de maneira ampla ${ }^{6}$. Foi incluída ao questionário a metodologia da EBIA, um instrumento de pesquisa que possibilita a estratificação dos sujeitos por níveis de insegurança alimentar, a qual foi cruzada com uma série de 


\section{ÍNDICE DE EFETIVIDADES DE POLÍTICAS PÚBLICAS DE SEGURANÇA ALIMENTAR PARA AGRICULTURA FAMILIAR NO SUDOESTE PAULISTA}

categorias de análise nas dimensões ambientais, sociais, econômicas e culturais, como características sociodemográficas (estrutura familiar, escolaridade), renda e consumo (renda individual e familiar, renda agrícola e não agrícola, renda agroindustrial, gasto com alimentação), condições de moradia, alimentação (perfil alimentar, forma de acesso aos alimentos e doenças associadas à alimentação), perfil da produção agrícola e agroindustrial, trabalho e lazer, redes de proteção social, autoconsumo, dentre outras. Foram realizadas 40 análises de variáveis simples e 25 cruzamentos.

Para a composição dos índices utilizou-se a técnica de análise fatorial. Segundo Johnson e Wichern (2008), a análise fatorial tem como principal objetivo descrever a variabilidade de um conjunto de dados utilizando um número menor de variáveis não observáveis, denominados fatores comuns. Esses fatores estão relacionados ao conjunto de dados por meio de um modelo linear, sendo que parte da variabilidade dos dados é atribuída aos próprios fatores e o restante atribuído às variáveis que não foram incluídas no modelo, ou seja, o erro aleatório. Em particular, o modelo da análise fatorial é dado por:

$$
(X-\mu)_{(p \times 1)}=L_{(p \times m)} * F_{(m \times 1)}+\varepsilon_{(p \times 1)}
$$

em que $\mu_{i}=$ média da variável $i, \varepsilon_{i}=i$-ésimo fator específico, $F_{j}=j$-ésimo fator comum e $l_{i j}=$ carga fatorial da $i$-ésima variável no $j$-ésimo fator.

Uma característica importante dessa análise é a rotação fatorial, que permite rotacionar os fatores em torno da origem até que alguma outra posição mais interessante seja alcançada. Para este fim, utilizou-se um tipo de rotação ortogonal, que mantém os eixos entre os fatores a $90^{\circ}$, denominado VARIMAX. Esse tipo de rotação se concentra na simplificação das colunas da matriz fatorial, ou seja, maximiza a soma de variâncias de cargas exigidas da matriz fatorial. Ainda de acordo com Johnson e Wichern (2008), essa abordagem busca a melhor rotação dos eixos de modo que a nova matriz de cargas fatoriais tenha o maior número de coeficientes nulos.

Para mensurar o nível de SA dos agricultores familiares foi utilizado o Índice UFSCar de SA para agricultores familiares que valida a metodologia da EBIA e avança no entendimento de que outras variáveis interferem nas condições de SAN, não sendo somente a renda (ALMEIDA et al., 2015; CARDOZO, 2016).

O índice "UFSCar" de SA pode significar um avanço metodológico na compreensão do gradiente de insegurança alimentar, pois considera as especificidades de uma realidade territorial rural a partir da EBIA do sujeito agricultor familiar (ALMEIDA et al., 2015). Para construção do Índice utilizouse a técnica de análise fatorial a partir de um conjunto de variáveis que tem aderência teórica e metodológica com a SA dos agricultores familiares: a renda familiar total (X1); diversificação da produção (X2): 0-não, 1-sim; nível de segurança alimentar segundo a escala EBIA (X3): 0-grave, 1- 
moderada, 2-leve, 3-segurança alimentar; participação em cooperativa (X4): 0-não, 1-sim; autoconsumo (X5): 0-não, 1-sim e porcentagem da renda agrícola para a renda total (X6): 0-0 a 25\%, 1-25 a 50\%, 2-50 a 75\%, 3-75 a 100\% (Almeida et al., 2015).

Visando a criação do índice de Índice de efetividade de Políticas Públicas de Segurança Alimentar (EPP), as seguintes variáveis foram consideradas: VAF5 - A família é beneficiária de algum programa: 0 - não, 1 -sim; VAF9 - Nível de segurança alimentar: 0 - grave, 1 - moderada, 2 - leve, 3 segurança alimentar; VAF19 - Renda total em R\$; VAF24 - Principal destino da produção (mercado institucional): 0 - não, 1 - sim; VAF26 - Uso de crédito para custeio ou financiamento nas últimas safras: 0 - não, 1 - sim; VAF27 - Recebimento de assistência técnica rural: 0 - não, 1 - sim; VAF28 Acessou o PAA: 0 - não, 1 - sim.

Foram consideradas as sete variáveis em estudo e todos os indivíduos que apresentaram valores válidos para as respectivas variáveis, formando um único conjunto de dados composto por 53 observações (17 indivíduos foram descartados por não satisfazerem a condição de valores válidos em todas as variáveis consideradas). O software utilizado para tratamento dos dados foi o software estatístico R. Feito isso, determinou-se o número de fatores a serem utilizados na análise por meio da proporção da variabilidade total explicada. Conforme a Tabela 2, pode-se observar que os três primeiros fatores explicam conjuntamente $60,26 \%$ da variabilidade total.

Tabela 2 - Valores dos autovalores, proporção de variância explicada e acumulada para cada um dos fatores obtidos

\begin{tabular}{|c|c|c|c|}
\hline Fator & Autovalor & $\begin{array}{l}\text { Proporção de Variância } \\
\text { Explicada }\end{array}$ & $\begin{array}{l}\text { Proporção de Variância } \\
\text { Explicada Acumulada }\end{array}$ \\
\hline 1 & 1,77 & 0,2526 & 0,2526 \\
\hline 2 & 1,29 & 0,1840 & 0,4366 \\
\hline 3 & 1,16 & 0,1660 & 0,6026 \\
\hline 4 & 1,04 & 0,1484 & 0,7510 \\
\hline 5 & 0,70 & 0,1002 & 0,8512 \\
\hline 6 & 0,60 & 0,0857 & 0,9369 \\
\hline 7 & 0,44 & 0,0630 & 1,0000 \\
\hline
\end{tabular}

Determinado o número de fatores, foi possível aplicar a rotação VARIMAX e obter as seguintes estimativas para obtenção dos escores fatoriais dos três fatores utilizados:

$$
\begin{gathered}
\text { Fator }_{1}=-0,2116 \times V A F 5^{*}+0,5437 \times V A F 9^{*}+0,3900 \times V A F 19^{*}+0,0380 \times V A F 24^{*} \\
+0,4194 \times V A F 26^{*}-0,0023 \times V A F 27^{*}-0,1423 \times V A F 28^{*} \\
\text { Fator }_{2}=0,0257 \times V A F 5^{*}-0,0846 \times V A F 9^{*}+0,1713 \times V A F 19^{*}+0,4817 \times V A F 24^{*} \\
-0,1595 \times V A F 26^{*}-0,2283 \times V A F 27^{*}+0,6005 \times V A F 28^{*} \\
\text { Fator }_{3}=0,6690 \times V A F 5^{*}-0,0416 \times V A F 9^{*}-0,2023 \times V A F 19^{*}+0,1184 \times V A F 24^{*} \\
+0,5361 \times V A F 26^{*}+0,1149 \times V A F 27^{*}-0,0050 \times V A F 28^{*}
\end{gathered}
$$




\section{ÍNDICE DE EFETIVIDADES DE POLÍTICAS PÚBLICAS DE SEGURANÇA ALIMENTAR PARA AGRICULTURA FAMILIAR NO SUDOESTE PAULISTA}

Calculou-se o valor do indicador através da ponderação dos escores fatoriais de cada um dos fatores pela proporção de variabilidade explicada pelo respectivo fator, após a rotação $(0,2134$ para o Fator 1, 0,21 para o Fator 2 e 0,1793 para o Fator 3):

$$
\begin{aligned}
\text { Indicador }= & 0,2134 \times \text { Fator }_{1}+0,21 \times \text { Fator }_{2}+0,1793 \times \text { Fator }_{3} \\
& \approx 0,08 \times V A F 5^{*}+0,09 \times V A F 9^{*}+0,08 \times V A F 19^{*}+0,13 \times V_{\text {VAF } 24^{*}} \\
& +0,15 \times V A F 26^{*}-0,03 \times V A F 27^{*}+0,09 \times V A F 28^{*}
\end{aligned}
$$

Os escores fatoriais foram obtidos através de cada um dos fatores utilizados, aplicando-se os valores padronizados (média 0 e variância 1) das variáveis que compõem o componente na própria fórmula do componente. Dessa forma, as variáveis $V A F 5^{*}, V A F 9^{*}, V A F 19^{*}, V A F 24^{*}, V A F 26^{*}$, $V A F 27^{*}$ e $V A F 28^{*}$ são respectivamente iguais a:

$$
V A F 5^{*}=\frac{V A F 5-M e ́ d i a(V A F 5)}{\text { Desvio Padrão(VAF5) }} \text { VAF9 } 9^{*}=\frac{V A F 9-\text { Média }(V A F 9)}{\text { Desvio Padrão(VAF9) }}
$$

E assim sucessivamente, sendo que:

Tabela 3 - Média de desvio padrão para as variáveis utilizadas

\begin{tabular}{lccccccc}
\hline & VAF5 & VAF9 & VAF19 & VAF24 & VAF26 & VAF27 & VAF28 \\
\hline Média & 0,86 & 2,65 & 1617,63 & 0,32 & 0,40 & 0,19 & 0,74 \\
Desvio Padrão & 0,35 & 0,61 & 1474,23 & 0,47 & 0,49 & 0,40 & 0,44 \\
\hline
\end{tabular}

Fonte: Autores (2018).

Aplicando a média e o desvio à fórmula, obteve-se:

$$
\begin{aligned}
& \text { Indicador }=0,08 \times\left(\frac{\text { VAF5 }}{0,35}\right)+0,09 \times\left(\frac{\text { VAF9 }}{0,61}\right)+0,08 \times\left(\frac{\text { VAF19 }}{1474,23}\right)+0,13 \times\left(\frac{\text { VAF } 24}{0,47}\right)+0,15 \times \\
& \left(\frac{\text { VAF } 26}{0,49}\right)-0,03 \times\left(\frac{\text { VAF27 }}{0,40}\right)+0,09 \times\left(\frac{\text { VAF28 }}{0,44}\right)-1,04
\end{aligned}
$$

Podemos observar, através dos coeficientes da fórmula do indicador, que as variáveis VAF24 - Principal destino da produção (mercado institucional) e VAF26 - Uso de crédito para custeio ou financiamento nas últimas safras apresentam os maiores pesos. Já as variáveis VAF5 - A família é beneficiária de algum programa, VAF9 - Nível de segurança alimentar, VAF19 - Renda total em R\$ e VAF28 - Acessou o PAA, apresentam pesos próximos. Com relação à variável VAF27 - Recebimento de assistência técnica rural, notamos que contribui negativamente para o indicador, devido ao sinal negativo, e também contribui muito pouco ao índice, uma vez que apresenta coeficiente próximo de zero.

Para efeito de comparação, calculou-se o valor máximo e mínimo do indicador simulando os valores para as respectivas variáveis que compõem 0 indicador através dos próprios dados utilizados. Dado $\mathrm{x}=$ valor do indicador e com os valores máximo e mínimo, podemos padronizar 0 indicador em uma escala de 0 a 100 através da seguinte transformação: 
Indicador $=\frac{x-\text { mínimo }}{\text { máximo-minimo }} \times 100$

Em que o máximo é dado por:

- VAF5 - A família é beneficiária de algum programa: 1 - sim;

- VAF9 - Nível de segurança alimentar: 3 - segurança alimentar;

- VAF19 - Renda total: $\mathrm{R} \$ 10.200,00$;

- VAF24 - Principal destino da produção (mercado institucional): 1 -sim;

- VAF26 - Uso de crédito para custeio ou financiamento nas últimas safras: 1 - sim;

- VAF27 - Recebimento de assistência técnica rural: 0 - não;

- VAF28 - Acessou o PAA: 1 - sim.

\section{Máximo $=1,01$}

E o mínimo dado por:

- VAF5 - A família é beneficiária de algum programa: 0 - não;

- VAF9 - Nível de segurança alimentar: 0 - grave;

- VAF19 - Renda total: $R \$ 402,00$;

- VAF24 - Principal destino da produção (mercado institucional): 0 - não;

- VAF26 - Uso de crédito para custeio ou financiamento nas últimas safras: 0 - não;

- VAF27 - Recebimento de assistência técnica rural: 1 - sim;

- VAF28 - Acessou o PAA: 0 - não.

Mínimo $=-1,08$

Portanto, aplicando-se os valores do máximo e do mínimo à transformação, temos:

$$
\begin{aligned}
& \text { Indicador }=\frac{100}{2,09} \times\left(0,08 \times\left(\frac{\text { VAF5 }}{0,35}\right)+0,09 \times\left(\frac{\text { VAF9 }}{0,61}\right)+0,08 \times\left(\frac{\text { VAF19 }}{1474,23}\right)+0,13 \times\left(\frac{\text { VAF } 24}{0,47}\right)+\right. \\
& \left.0,15 \times\left(\frac{\text { VAF26 }}{0,49}\right)-0,03 \times\left(\frac{\text { VAF27 }}{0,40}\right)+0,09 \times\left(\frac{\text { VAF28 }}{0,44}\right) \times+0,05\right) .
\end{aligned}
$$

Dessa forma, as seguintes classificações para o indicador podem ser atribuídas:

$0-20$ : Muito baixo

20,1 - 40: Baixo

40,1 - 60: Moderado

60,1 - 80: Alto

80,1 - 100: Muito Alto 
Para determinar o número de fatores a serem utilizados na composição do índice, levouse em consideração a proporção da variabilidade total explicada, com valor mínimo necessário igual a $50 \%$.

\section{RESULTADOS}

\section{1 Índice de efetividades das políticas públicas para os agricultores Familiares a partir das variáveis analíticas}

Aqui, o objetivo foi identificar quais políticas públicas têm efetividades para as questões produtivas, econômicas e relativas ao modo de vida e à organização social dos agricultores familiares. Trata também das relações entre as mesmas que explicam a SA a partir do índice UFSCar, abrindo veredas analíticas para a construção do novo índice de efetividades e as correlações com o índice de segurança alimentar (ALMEIDA et al., 2015). Como foi visto anteriormente, a renda familiar, a inclusão da pluriatividade econômica do produtor, assim como a sua participação enquanto ator produtivo em organizações sociais, o grau do autoconsumo familiar praticado e as diversificações de produção foram as variáveis escolhidas pelas suas aderências teóricas e metodológicas escolhidas analiticamente, sendo realizadas análises dos cruzamentos destas variáveis com a EBIA. Isso permite melhor compreensão e aderência do desenvolvimento da SA dos agricultores familiares territoriais (ALMEIDA et al., 2015).

Os resultados obtidos por meio da variável de participação em programas de SAN no território (PNAE, PAA, dentre outros) demonstram efeitos significativos na SA dos agricultores beneficiários em detrimento aos não beneficiários. A Tabela 4 mostra que os agricultores beneficiários têm grau moderado de insegurança alimentar e um índice 37\% maior dos agricultores não beneficiários, que se classificaram em baixa SA.

Tabela 4 - Índice de efetividade de políticas públicas e se a família é beneficiária de algum programa de segurança alimentar

\begin{tabular}{ccccc}
\hline & N & Indicador & \multicolumn{2}{c}{ Classificação } \\
\hline Não & 8 & 39,37 & Baixo \\
\hline Sim & 49 & 53,78 & Moderado \\
\hline
\end{tabular}

Fonte: Autores (2018).

Os resultados também corroboram com a metodologia em relação às diferentes categorias de insegurança alimentar dos agricultores e às efetividades das políticas públicas em que são sujeitos, 
como demonstra a Tabela 5. Agricultores com algum grau de insegurança alimentar (leve - IAL, moderada - IAM ou grave- IAG) apresentam baixo índice de efetividades de políticas públicas; em contrapartida, os agricultores em estágio de SA se apresentam como moderada efetividade e num índice quantitativo $43 \%$ superior à IAL, que se expressa em mais agricultores entrevistados.

Constata-se que o índice de efetividade de políticas públicas tem aderência ao índice de segurança EBIA, largamente validado e utilizado em agências oficiais e trabalhos científicos. Esses resultados têm extrema importância para a validação dos índices apresentados neste trabalho, em relação não ambígua de efetividades de políticas públicas com SA dos agricultores beneficiários em diferentes metodologias (EBIA e índice UFSCar).

Tabela 5 - Índice de efetividade de políticas públicas e nível de SA pela EBIA

\begin{tabular}{ccccc}
\hline & $\mathbf{N}$ & Indicador & Classificação \\
\hline Grave & 1 & 35,48 & Baixo \\
\hline Moderada & 1 & 32,38 & Baixo \\
\hline Leve & 15 & 39,78 & Baixo \\
\hline $\begin{array}{c}\text { Segurança } \\
\text { Alimentar }\end{array}$ & 40 & 57,14 & Moderado \\
\hline Fonte: Autores (2018) & &
\end{tabular}

Fonte: Autores (2018).

A partir da constatação anterior, os resultados da Tabela 6 são relevantes para compreender como e quantas variáveis analíticas de canais de comercialização, utilização de créditos agrícolas, assistência técnica rural e acesso ao PAA interferem nos resultados do índice de efetividades

Contata-se que os agricultores com inserção de seus produtos nos mercados institucionais a partir das políticas públicas de SAN, principalmente o PAA, têm ganhos significativos de efetividades. Aponta que os mercados institucionais são estruturantes para reprodução econômica da agricultura familiar e que o PAA é um instrumento muito efetivo e dinamizador das políticas de SAN no Brasil. Outro elemento importante para o índice é o uso de crédito e financiamento via principalmente 0 Programa Nacional da Agricultura Familiar (Pronaf) que se apresenta com condições de acesso e pagamentos bastante favoráveis aos praticados no mercado e incentiva a utilização de insumos mais agroecológicos e de novas tecnologias.

Finalmente, os dados mostram a baixa efetividade da política de Ater. Isso alerta para a necessidade de reconstrução da agenda, desse importante instrumento para os agricultores familiares. 
Tabela 6 - Índice de Efetividade e variáveis analíticas de produção, crédito e de políticas públicas

\begin{tabular}{ccc}
\hline Variáveis & Índice(\%) & Classificação \\
\hline $\begin{array}{c}\text { Principal destino da produção - Mercado } \\
\text { Institucional }\end{array}$ & & \\
Não & 44,29 & Moderado \\
Sim & 67,93 & Alto
\end{tabular}

$\begin{aligned} & \text { Uso de crédito para custeio e } \\
& \text { financiamento nas últimas safras }\end{aligned}$
\begin{tabular}{lll} 
Não & 42,45 & Moderado \\
$\operatorname{Sim}$ & 65,52 & Alto \\
\hline
\end{tabular}

Recebimento de assistência técnica rural

\begin{tabular}{ccc} 
Não & 53,12 & Moderado \\
Sim & 46,04 & Moderado \\
\hline $\begin{array}{c}\text { Utilização de insumos ou novas } \\
\text { tecnologias } \\
\text { Não }\end{array}$ & 43,20 & Moderado \\
Sim & 53,52 & Moderado \\
\hline Se acessou o PAA & & Baixo \\
Não & 37,74 & Moderado \\
\hline
\end{tabular}

Fonte: Autores (2018).

\subsection{Relação entre os índices de SA e de EPP}

A fim de possibilitar a interpretação da relação entre SA e EPP em termos de elasticidades, foi realizada uma regressão linear por Mínimos Quadrados Ordinários (MQO) com as variáveis em logaritmos naturais, e resíduos robustos à heteroscedasticidade, visto que o teste de Breusch-Pagan resultou em um p-valor $<0.001$, tal qual apresentado na Tabela 7 , a qual demonstra que a variável In_EPP é estatisticamente significante. 
Tabela 7 - Regressão linear

\begin{tabular}{cccccc}
\hline Variável & Coef. & $\begin{array}{c}\text { Robust } \\
\text { Std. Err. }\end{array}$ & $\mathrm{P}>|\mathrm{t}|$ & \multicolumn{2}{l}{ [95\% Conf. Interval] } \\
& & & & & \\
\hline In_EPP & 0.5093 & 0.147428 & 0.001 & .2130331 & .8055663 \\
_Cons & 1.809568 & 0.598664 & 0.004 & .6065087 & 3.012628 \\
\hline
\end{tabular}

Fonte: Autores (2018).

Tal relação pode ser mais bem visualizada por meio da Figura 1, a qual apresenta 0 scatterplot das variáveis SA e EPP (ambas em logaritmos naturais), bem como a reta de regressão e seu intervalo de confiança com significância de $5 \%$. Tais resultados demonstram que uma elevação de $1 \%$ no EPP acarreta em um incremento de aproximadamente $0,51 \%$ no índice de SA para a agricultura familiar.

Figura 1 - Relação entre SA e EPP

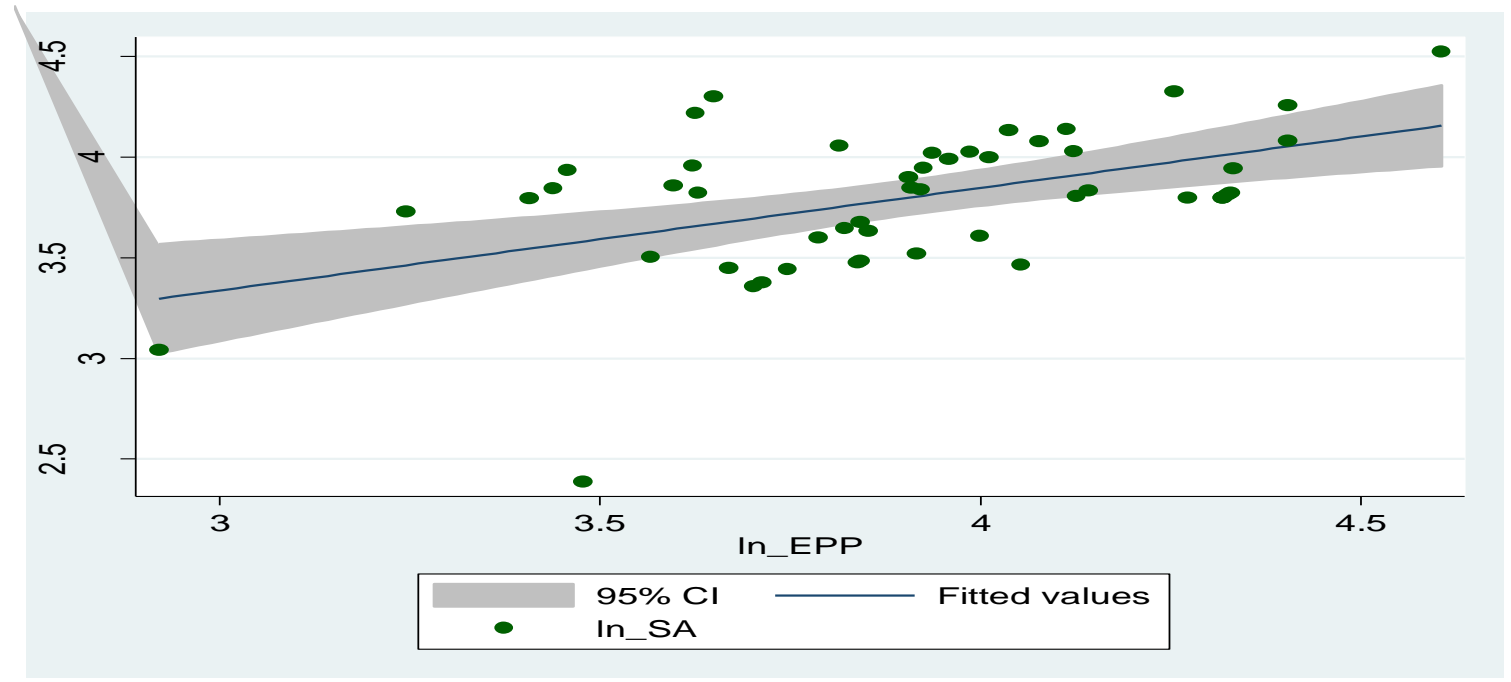

Notas: $\mathrm{Cl}$ - Intervalo de confiança; In_SA - índice SA; Fitted values- valores ajustados Fonte: Autores (2018).

Uma nova regressão linear por MQO robusta à heteroscedasticidade (teste de BreuschPagan com $p<0,001$ ) foi realizada a fim de explorar a influência das variáveis de EPP sobre a SA . A Tabela 8 demonstra que as variáveis VAF9 (Caracterização da Segurança Alimentar-EBIA), VAF19 (Renda Total Familiar), e VAF24 (Principal Destino da Produção ser Mercado Institucional) apresentam elasticidades positivas e significantes, indicando para a variável VAF19, por exemplo, que um incremento de $1 \%$ na Renda total acarreta uma elevação de $0,26 \%$ no indicador de SA. De forma 
similar, os indivíduos com acesso ao mercado institucional possuem um nível de SA 15\% acima da média.

Tabela 8 - Regressão linear: Influência das variáveis de EPP sobre a SA

\begin{tabular}{cccccc}
\hline Variável & Coef. & $\begin{array}{c}\text { Robust } \\
\text { Std. Err. }\end{array}$ & $\mathbf{P}>\mathbf{| t |}$ & {$[95 \%$ Conf. Interval] } \\
\hline VAF5 & 0.077 & 0.144 & 0.596 & -.2137365 & .3676861 \\
VAF9 & 0.337 & 0.107 & 0.003 & .1212673 & .553585 \\
VAF19 & 0.260 & 0.044 & 0.000 & .171255 & .3491864 \\
VAF24 & 0.154 & 0.075 & 0.045 & .0032327 & .3053101 \\
VAF26 & -0.041 & 0.066 & 0.536 & -.1730033 & .0912401 \\
VAF27 & 0.087 & 0.093 & 0.356 & -.1009618 & .2746074 \\
VAF28 & -0.129 & 0.083 & 0.127 & -.2966533 & .0382829 \\
_Cons & 1.019 & 0.514 & 0.054 & -.016749 & 2.055508 \\
\hline
\end{tabular}

Fonte: Autores (2018).

\section{CONCLUSÃO}

A incorporação do problema da SAN no mundo abriu uma agenda de desafios que apenas começou no final dos anos 80, mais intensamente a partir de 1986, com a conferência mundial da FAOONU em Roma. O conceito de SAN desenvolvido a partir dali ofereceu um conjunto significativo de elementos e questões que podem ser desenvolvidos no campo das políticas públicas. Agricultura familiar tem sido um objeto importante para políticas públicas de SAN. Descritores do nível de SA da agricultura familiar representam um dos desafios na atualidade e que o presente trabalho pretendeu avançar.

Na perspectiva de construção de um novo índice de EPP de SAN para agricultores familiares no Brasil, o presente trabalho considerou pesquisar alguns descritores de SA para agricultura familiar na região sudoeste do Estado de São Paulo. Utilizando a técnica de análise fatorial, a partir de um conjunto de variáveis que têm aderência teórica e metodológica com a SA dos agricultores familiares, pressupondo que a EBIA possui limitações analíticas, o trabalho sobre o índice UFSCar de EPP de SA para a agricultura familiar apresentou algumas constatações.

Uma elevação de 1\% no índice de EPP acarreta um incremento de aproximadamente 0,51\% no índice de SA para a agricultura familiar. Já o acréscimo de 1\% na renda total acarreta uma elevação de $0,26 \%$ no indicador de SA. De forma similar, os indivíduos com acesso ao mercado 
institucional possuem um nível de SA de 15\% acima da média. Isso mostra que, na região sudoeste do Estado de São Paulo, os mercados institucionais são estruturantes para a reprodução econômica da agricultura familiar e que o PAA tem relativa eficácia na SAN no Brasil.

A utilização de crédito e financiamento por meio do Pronaf mostrou-se importante para 0 índice EPP, já que apresentou condições de acesso e pagamentos bastante favoráveis aos praticados no mercado (com percentual de 65,52\%), além de incentivar a utilização de insumos mais agroecológicos e de novas tecnologias.

O mesmo não aconteceu com a política de Ater, cuja efetividade não foi significativa neste estudo, apresentando uma baixa utilização pelos agricultores (apenas $53,12 \%$ ), e, portanto, não acarretando melhorias dos níveis de SA dos mesmos.

Com isso, comprova-se a hipótese de que a efetividade é positiva para o agricultor que é beneficiário de um programa de SA, como o PAA, que tem acesso a créditos via Pronaf, que possui como principal canal de comercialização o mercado institucional, e que tem renda agrícola especializada, em contrapartida, que recebe Ater. Dessa forma, a aplicação e a análise do índice UFSCar de políticas públicas territoriais de SAN desenvolvidas na região sudoeste do Estado de São Paulo, mostra ser possível também comparar impactos para agricultores familiares em regiões distintas no Brasil.

Sabe-se da relevante heterogeneidade estrutural e regional da economia e da sociedade brasileira. Entretanto, os indicadores utilizados em uma região do Estado de São Paulo representam contribuição importante para apreciação de efetividades de políticas públicas de SAN no Brasil, independentemente do nível de desenvolvimento econômico, social e de maturidade desse tipo de política pública.

\section{REFERÊNCIAS}

ALMEIDA, L. M. M. C. Estrutura de Governança e Gestão das Redes e Programas de Segurança Alimentar: Análise Comparativa entre Municípios Paulistas (Pós-Doutorado). Universidade Estadual de Campinas, 2009.

ALMEIDA, L.M.M.C. et al. Índice UFSCar de Efetividades do Programa de Aquisição de Alimentos para a segurança alimentar e nutricional de agricultores familiares do interior paulista. Gestão \& Produção, v. 25, p. 500-512, 2018.

ALMEIDA, L. M. M. C. et al. Índice UFSCar de segurança alimentar para agricultores familiares.

Revista de Política Agrícola, v. 24, n. 4, p. 82-96, 2015. 
BELLONI, I.; MAGALHÃES, H.; SOUSA, L.C. Metodologia para avaliação de políticas públicas: uma experiência em educação profissional. São Paulo: Cortez, 2001. 96 p. (Coleção Questões da Nossa Época, v. 75).

BUCCI, M. P. D. Direito administrativo e políticas públicas. São Paulo: Saraiva, 2002.

CARDOZO, D. R. Renda, consumo alimentar e estado nutricional como indicadores complementares à insegurança alimentar e nutricional do Programa Bolsa Família (Tese de Doutorado). Araraquara: Universidade Estadual Paulista, 2016.

COCHRAN, W. G. Sampling techniques. Nova York: J. Wiley, 1953.

HALL, P.; TAYLOR, R. As três versões do neo-institucionalismo. Lua Nova, São Paulo, n. 58, p.193223, 2003.

INSTITUTO BRASILEIRO DE GEOGRAFIA E ESTATÍSITCA (IBGE). Dados sobre agricultura familiar. 2010. Disponível em: https://www.ibge.gov.brl. Acesso em: jan. 2019.

JOHNSON, R. A.; WICHERN, D. W. Applied multivariate statistical analysis. 6. ed. Upper Saddle River: Prentice Hall, 2008.

PAULILLO, L. F.O.; PESSANHA, L. Segurança alimentar, políticas públicas e regionalização. In. PAULILLO, L. F.O. et. al. Reestruturação agroindustrial, políticas públicas e segurança alimentar regional. São Carlos: Edufscar, 2002.

SEGALL-CORREAA, A. M.; MARIN-LEON, L. A Segurança Alimentar no Brasil: Proposição e Usos da Escala Brasileira de Medida da Insegurança Alimentar (EBIA) de 2003 a 2009. Segurança Alimentar e Nutricional. Campinas, SP, v. 16, n. 2, p. 1-19, 2009.

SILVA FILHO, A. Metodologias de avaliação de programas público em Nutrição: a análise e aplicação aos programas brasileiros (Dissertação de Mestrado). São Paulo: USP, 2005.

SILVA, N. N. Amostragem probabilística: um curso introdutório. 2. ed. São Paulo: Edusp, 2001. 124 p. (Acadêmica, 18).

SOUZA, C. Políticas públicas: uma revisão da literatura. Sociologias. Porto Alegre, RS, v. 8, n. 16, p. 20-45, 2006.

\section{Notas}

1 Espaço que ganhou recentemente um novo campus da UFSCar, na Fazenda Lagoa do Sino no município de Buri - SP. Constatou-se que o conjunto de municípios deste território de desenvolvimento rural foi escolhido pelo parâmetro de proximidade ao Campus e apresenta os menores índices de desenvolvimento do Estado de São Paulo, tanto pela metodologia do IDH Municipal (IDH-M) quanto pela metodologia do Índice Paulista de Responsabilidade Social (IPRS). 0 Território Lagoa do Sino é composto por 40 municípios sendo 18 pertencentes à microrregião de Itapetininga, 13 à microrregião de Itapeva e 9 à microrregião de Avaré.

2 De acordo com Segall-Corrêa e Marin-León (2009), a EBIA é um método de mensuração da situação alimentar domiciliar que objetiva, a partir da percepção do sujeito, captar distintas dimensões da Insegurança Alimentar (IA), as quais variam de Segurança Alimentar (SA) - quando não há restrição alimentar de qualquer natureza, nem mesmo a preocupação com a falta de alimento no futuro - até a Insegurança Alimentar Grave (IAG) - deficiência quantitativa e com alta possibilidade de 
fome entre adultos e crianças da família. Entre os dois extremos estão os níveis de Insegurança Alimentar Leve (IAL) quando a alimentação é afetada juntamente com a preocupação de que possam faltar alimentos num futuro próximo - e a Insegurança Alimentar Moderada (IAM) - quando começa haver restrição quantitativa na alimentação dos adultos da família.

${ }^{3}$ Um território que contém 40 municípios com os menores IDH do Estado de São Paulo.

${ }^{4} \mathrm{~A}$ elaboração do índice considera um consórcio de 15 municípios e não se limita a ele porque o IDH ainda é um sinalizador inicial fundamental para os estudos locais das dificuldades e da SAN de agricultores familiares por conter indicadores clássicos de riqueza, educação, saúde, etc.

${ }^{5}$ Visão da política pública como um processo incremental, baseada no empirismo e visão de que políticas passadas influenciam nas decisões futuras dos governos (SOUZA, 2006).

${ }^{6}$ Elementos de SA tratada de maneira ampla abarcam variáveis ou indicadores econômicos, sociais, culturais e ambientais como: nível de segurança alimentar (EBIA), saúde, escolaridade, redes de cooperação, trabalho, renda, pluriatividade, formação de redes de proteção social, autoconsumo, práticas ambientais mais sustentáveis, acesso ao crédito, dentre outros (ALMEIDA, 2009). 